\title{
Impaired antioxidant defence and accumulation of oxidative stress in caspase-2-deficient mice
}

\author{
S Shalini ${ }^{1}$, L Dorstyn ${ }^{1,2}$, C Wilson ${ }^{1}$, J Puccini ${ }^{1,2}$, L Ho ${ }^{1}$ and S Kumar ${ }^{\star, 1,2}$
}

Caspase-2 has been implicated in apoptosis and in non-apoptotic processes such as cell cycle regulation, tumor suppression and ageing. Using caspase-2 knockout $\left(\operatorname{casp2}^{-l}\right)$ mice, we show here that the putative anti-ageing role of this caspase is due in part to its involvement in the stress response pathway. The old casp2 ${ }^{-I-}$ mice show increased cellular levels of oxidized proteins, lipid peroxides and DNA damage, suggesting enhanced oxidative stress. Furthermore, murine embryonic fibroblasts from casp $^{-l-}$ mice showed increased reactive oxygen species generation when challenged with pro-oxidants. Reduced activities of antioxidant enzymes glutathione peroxidase (GSH-Px) and superoxide dismutase (SOD) were observed in the old casp2 $^{-I-}$ mice. Interestingly, in the old casp2 ${ }^{-I-}$ animals expression of FoxO1 and FoxO3a was significantly reduced, whereas p21 levels and the number of senescent hepatocytes were elevated. In contrast to young wild-type mice, the casp2 ${ }^{-I-}$ animals fed an on ethanol-based diet failed to show enhanced GSH-Px and SOD activities. Thus, caspase-2, most likely via FoxO transcription factors, regulates the oxidative stress response in vivo.

Cell Death and Differentiation (2012) 19, 1370-1380; doi:10.1038/cdd.2012.13; published online 17 February 2012

Caspase-2 belongs to a family of cysteine aspartate proteases that are involved in the execution of apoptosis and in non-apoptotic processes, such as cytokine processing, cell cycle regulation, tumor suppression and ageing. ${ }^{1}$ It is one of the first discovered and evolutionarily most conserved among caspases. ${ }^{2-4}$ Many in vitro studies suggest a role for caspase-2 in DNA damage and stress-induced cell death, although mice lacking caspase-2 show no overt phenotype and reproduce normally. ${ }^{5,6}$ Interestingly, caspase-2 knockout $\left(\right.$ casp $\left.^{-/-}\right)$animals develop signs of early onset ageing compared with their wild-type counterparts, and this was primarily attributed to their inability to eliminate damaged cells in the absence of caspase-2. ${ }^{7}$ However, the mechanism by which caspase- 2 prevents such an accumulation of damaged cells is not known and it is unclear whether this role of caspase-2 is dependent on its function in apoptosis or in non-apoptotic pathways.

Recent work has revealed that caspase-2 acts as a suppressor of Myc-induced lymphomagenesis. ${ }^{8}$ There is also emerging evidence supporting a role for caspase- 2 in preventing oncogene-induced cell transformation and tumorigenesis, which further implicates caspase-2 in cell cycle checkpoint maintenance, preventing aberrant cell division and aneuploidy. ${ }^{2,8}$ Under stress conditions and even during an unperturbed cell cycle, checkpoint proteins have a key role in genome maintenance by mediating cellular responses to DNA damage, and represent an essential part of the cellular stress response. Maintenance of genome integrity in the face of such events is essential to prevent early-onset of ageing. Loss of many cell cycle and DNA repair genes cause premature ageing. For example, mice with defective nucleotide excision repair (trichothiodystrophy and Cockayne syndrome) and ataxia telangiectasia- and Rad3-related (ATR) deficiency show premature ageing. ${ }^{9}$

Ageing is a process associated with the accumulation of cellular damage and a progressive decline in organ function and repair ability. Several theories have been put forward to explain this phenomenon, including oxidative damage to cells as the most common cause of ageing. Oxidative stress arises when there is an excessive generation of reactive oxygen species (ROS) such as oxygen ions or peroxides, or reduction in the free radical scavenging machinery such as loss of antioxidant enzymes or other antioxidants. ${ }^{10}$ ROS are normal by-products of cellular metabolism and have important roles in cell signalling and cellular homeostasis. Excessive ROS causes damage to DNA, RNA and protein, which are factors contributing to the physiology of ageing and cancer. The ROSinduced cellular stress response is controlled at the molecular level by highly conserved signalling molecules including proteins involved in the insulin/insulin like growth factor (IGF) signalling, sirtuins (SIR), target of rapamycin (TOR) and AMP-activated protein kinase (AMPK) pathways, ${ }^{11}$ which converge on the regulatory proteins p53 and FoxO. Recent studies indicate that caspase- 2 regulates $p 53^{8,12}$ well-known for its role in tumor suppression, DNA damage, oxidative stress and ageing. ${ }^{13}$ Among its targets are several antioxidant enzymes and genotoxic stress-induced proteins of the sestrin family (Sesn1-3). ${ }^{13}$

FoxO family proteins are a subgroup of the forkhead family of transcription factors. ${ }^{14,15}$ Mammalian FoxO members,

\footnotetext{
${ }^{1}$ Centre for Cancer Biology, SA Pathology, Adelaide, SA, Australia and ${ }^{2}$ Department of Medicine, University of Adelaide, Adelaide, SA, Australia ${ }^{*}$ Corresponding author: S Kumar, Centre for Cancer Biology, SA Pathology, PO Box 14, Rundle Mall, Adelaide, SA 5000, Australia. Tel: +61 882223738 ; Fax: + 618 82223162; E-mail: sharad.kumar@health.sa.gov.au

Keywords: oxidative stress; ROS; antioxidant; caspases; ageing

Abbreviations: ROS, reactive oxygen species; Casp2, caspase-2; FoxO, forkhead box class 0; GSH-Px, glutathione peroxidase; MEF, murine embryonic fibroblast; 8-OHdG, 8-hydroxydeoxyguanosine; Sesn, sestrins; SOD, superoxide dismutase; TUNEL, terminal deoxynucleotidyl transferase dUTP nick end labelling Received 16.1.12; revised 23.1.12; accepted 25.1.12; Edited by G Melino; published online 17.2.12
} 
including FoxO1, FoxO3a, FoxO4 and FoxO6 are involved in multiple biological processes, such as cell-cycle arrest, DNA repair, apoptosis, glucose metabolism, ageing and autophagy. ${ }^{14,15}$ FoxO proteins also regulate the transcription of several antioxidant genes including superoxide dismutase 2 (SOD2), catalase and sestrins and has been linked to tumor suppression ${ }^{16-18}$ and longevity pathways. ${ }^{14}$ As such, examining these regulatory pathways is crucial for understanding the early ageing phenotype in the caspase- $2^{-1-}$ animals. In this study, we provide evidence that the loss of caspase-2 increases oxidative stress in vivo by altering these regulatory proteins and the cellular stress response pathways.

\section{Results}

Casp2 $^{-1-}$ mice display premature ageing-related traits. Loss of caspase-2 has been reported to be associated with early onset ageing in mice. ${ }^{7}$ A comparison of the survival curves showed no significant difference in the median lifespan of casp $^{-/-}$and wild-type $\left(\right.$casp $\left.^{+/+}\right)$ animals (Supplementary Figure S1). The median lifespan of casp2 $^{-1-}$ mice was about 730 days compared with 760 days for the wild-type mice $(P=0.08$, Table 1). However, as previously reported in an independently generated line of knockout mice, ${ }^{7}$ the maximum lifespan of casp2 $^{-1-}$ mice was significantly shorter $(P=0.0465$, Fisher's exact test) (Table 1; Supplementary Figure S1a). None of the casp2 ${ }^{-1-}$ animals survived beyond 850 days, whereas $5 / 18$ wild-type $(27 \%)$ animals lived beyond this time (Supplementary Figure S1). Furthermore, when comparing the survival curves for the last $50 \%$ survivors, a significant difference in the lifespan was noticed ( $P=0.0325$, Fisher's exact test) (Supplementary Figure S1b). The casp2 $2^{-1-}$ mice show some signs of early ageing such as early hair greying and hair loss. Comparison of other age-related features revealed significantly reduced body weight and bone volume in the old casp $^{-1-}$ mice (Table 1). No difference in the incidence of cataracts or spontaneous tumor development was observed in the old casp2 ${ }^{-1-}$ mice. Also, there were no significant differences in the incidence of lymphocytic infiltrates in livers of old casp2 $2^{-1-}$ mice compared to wild-type mice at 24-26 months (Table 1). Analysis of skin histology from crosssections of dorsal skin from 24-month-old wild-type and casp2 $2^{-1-}$ mice showed a striking reduction in subcutaneous

Table 1 Age-associated phenotypes in $\mathrm{Casp}^{+/+}$and $\mathrm{Casp2}^{-/-}$mice at 24 months of age

\begin{tabular}{lcc}
\hline & Casp2 $^{+/+}$ & Casp2 $^{-/}$ \\
\hline Median lifespan (days) & 760 & 730 \\
Maximum lifespan (days) & 912 & $850^{*}$ \\
Body weight (g) & $35.93 \pm 2.76$ & $31.8 \pm 3.83^{* *}$ \\
Bone volume (mm ${ }^{*}$ ) & $1.4 \pm 0.19$ & $0.9 \pm 0.17^{\star *}$ \\
Hair greying/alopecia & $4 / 18$ & $10 / 19$ \\
Lymphoid infiltrates (liver) & $4 / 10$ & $8 / 10$ \\
Splenomegaly & $0 / 18$ & $6 / 19$ \\
Tumor incidence & $4 / 18$ & $2 / 19$ \\
Blood chemistry & Normal & Normal
\end{tabular}

Values are mean \pm S.D. ( $n=18$ for Casp $^{+/+}$or 19 for Casp $^{-/-}$for survival analysis $)$ ${ }^{\star} P<0.05,{ }^{\star \star} P<0.002$ represents a comparison between the two genotype groups adipose tissue (SAT) in both male and female animals (Supplementary Figure S2a and S2b). In addition, while we did not observe a decrease in the muscle fiber diameter, there was a significant reduction in the muscle mass in old casp2 $^{-/-}$male mice (Supplementary Figure S2b). The epidermal layer was also significantly thinner in the female casp2 $2^{-1-}$ mice but not in male mice (data not shown). We did not observe any differences in SAT or the muscle layer in younger mice, indicating that it reflects an age-dependent process. Consistent with earlier studies, ${ }^{7}$ a shorter maximum lifespan, reduced body weight, reduced fat content and low bone volume in the casp2 ${ }^{-1}$ animals suggests a trend towards early ageing in these mice.

Caspase-2 deficiency leads to increased oxidative stress. Oxidative stress is caused by the accumulation of ROS in the cell. As the oxidative stress is often associated with premature ageing, we assessed whether the caspase-2deficient mice displayed increased levels of ROS. For studies reported here the animals were monitored for 24-26 months (old mice), while animals at 2-3 months were used as young mice. Damage caused by free radicals was determined by measuring lipid peroxidation and protein carbonyls, which were both significantly increased in the old casp2 ${ }^{-1-}$ mice compared with the old wild-type animals (Figures 1a and b). The activity of antioxidant enzyme SOD was reduced in the old casp2 ${ }^{-1-}$ mice (Figure 1c). Glutathione peroxidase (GSH-Px) activity was also significantly decreased in these mice (Figure 1d). No difference was noticed in the young (2-3 month old) mice, although protein carbonyl content and SOD activity were higher in casp2 $2^{-1-}$ animals, indicating that younger animals do not present an abnormal oxidative stress response (Supplementary Figure S3). This suggests a compromised antioxidant defence system and accumulation of free radicals in ageing caspase-2-deficient animals.

To further validate this in vitro, we used immortalized murine embryonic fibroblasts (MEFs) from casp2 $^{-1}$ and wild-type mice, and treated cells with the ROS generator menadione, followed by staining with dichlorofluoroscein diacetate (CM-H2DCFDA). Casp2 ${ }^{-1-}$ MEFs showed significantly higher fluorescence indicative of increased ROS generation compared with the wild-type MEFs (Figure 1e). These data indicate that the loss of caspase-2 is associated with an impaired antioxidant defence system.

Increased DNA damage in casp2 ${ }^{-1-}$ mice. ROS-induced oxidative damage includes DNA strand breaks and nucleotide modifications, which trigger a DNA damage response, that is mediated by the recruitment of ATM and ATR to the site of DNA damage and subsequent phosphorylation of Ser-139 of H2AX $(\gamma-\mathrm{H} 2 \mathrm{AX}) .{ }^{19}$ This facilitates the assembly of multi-protein repair complexes to the site of DNA damage. As such $\gamma-\mathrm{H} 2 \mathrm{AX}$ foci mark potential DNA damage sites and $\gamma-\mathrm{H} 2 \mathrm{AX}$ immunohistochemistry has been established as a reliable marker to measure DNA damage. $^{20}$ We observed an increase in the number of $\gamma-\mathrm{H} 2 \mathrm{AX}$ positive hepatocytes in liver sections of old casp2 $2^{-1-}$ mice (Figures $2 a$ and $b$ ). Oxidative damage to DNA was also measured by quantitation of 8-hydroxydeoxyguanosine (8-OHdG), an oxidatively modified base. No difference in 

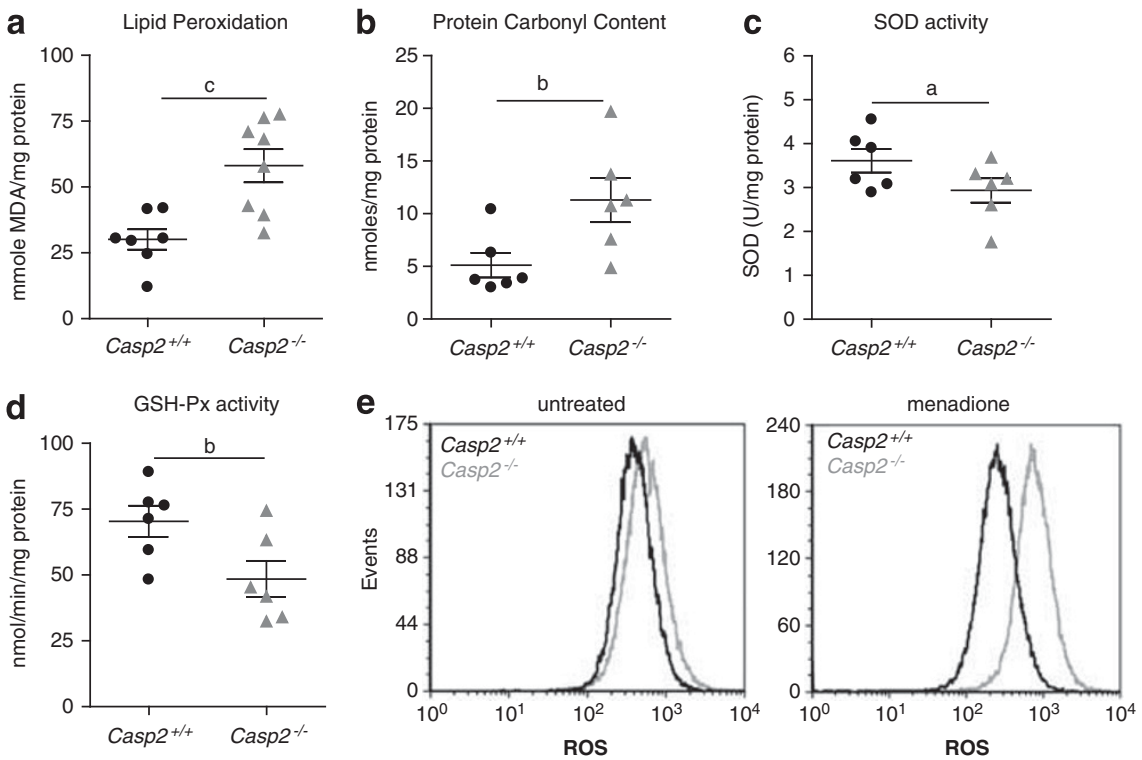

Figure 1 Loss of caspase-2 increases oxidative stress. Increased (a) lipid peroxidation, (b) protein oxidation levels and reduced (c) SOD activity, (d) GSH-Px activity in old Casp2 $^{-1-}$ mice liver. Each dot or triangle represents an individual animal. Values are mean \pm S.E.M. for six to seven animals per group. ${ }^{\mathrm{a}} P<0.05,{ }^{\mathrm{b}} P<0.01$ and ${ }^{\mathrm{c}} P<0.001$ represent comparison between the two genotypes. (e) ROS levels in untreated and menadione treated SV40 immortalized MEFs. Results shown are representative of three independent experiments
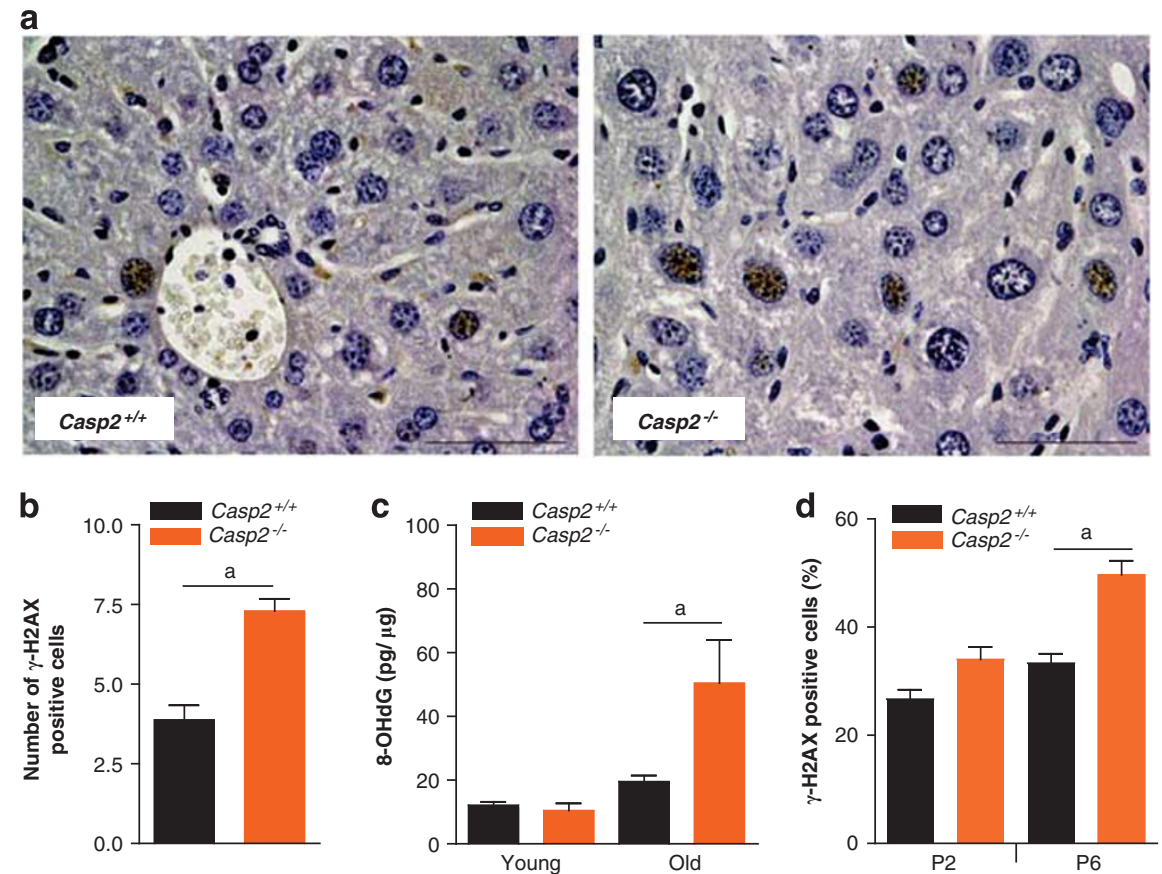

Figure 2 Old Casp2 ${ }^{-1-}$ mice display increased DNA damage. (a) Immunohistochemical localization of $\gamma-\mathrm{H} 2 \mathrm{AX}$ in aged mice liver $(\times 40)$, scale bar $=50 \mu \mathrm{m}$. (b) Quantitation of $\gamma-\mathrm{H} 2 \mathrm{AX}$ stained cells in liver sections from old Casp2 ${ }^{+/+}$and $\mathrm{Casp2}^{-1-}$ mice. Values are average number of $\gamma$-H2AX positive cells per field of view at $\times 40$ magnification. (c) 8-OHdG levels in liver DNA from young and old Casp2 ${ }^{+/+}$and Casp2 ${ }^{-1-}$ animals ( $n=5$ or 4 ) were determined by using an EIA kit (Cayman Chemicals). Values are mean \pm S.E.M. ${ }^{a} P<0.05$ represents comparison between the two groups. (d) Casp2 ${ }^{-1}$ MEFs at early passage (P2) and late passage (P6) stained with $\gamma-\mathrm{H} 2 \mathrm{AX}$ after menadione treatment and the frequency of $\gamma$-H2AX positive cells quantitated. Values are mean \pm S.E.M. ${ }^{a} P<0.05$ represents comparison between the groups

8-OHdG levels was observed in the young mice. However, with advancing age, wild-type and casp2 $^{-1-}$ mice both showed significant increase in 8-OHdG levels. Again we observed higher levels of 8-OHdG in the old casp2 $2^{-1-}$ mice compared with the wild-type animals (Figure 2c).
We used primary MEFs, treated with menadione to induce ROS in vitro, and also found that the frequency of $\gamma-\mathrm{H} 2 \mathrm{AX}$ foci in menadione-treated casp2 ${ }^{-1-}$ MEFs was significantly higher particularly in later passage (P6) MEFs, which commonly display higher oxidative stress through culturing (Figure 2d). 
This again indicates that absence of caspase-2 increases the accumulation of DNA damage following ROS induction.

Old casp2 $^{-1-}$ mice show increased senescent cells in liver. In order to further characterize the type of damage caused by ROS, we assessed cellular senescence by $\beta$-galactosidase $(\beta$-gal) staining of liver sections from old animals. We quantitated the frequency of $\beta$-gal positive hepatocytes in these liver sections and found a significant increase in casp $^{-1-}$ mice (Figure 3a). Although loss of caspase-2 has been shown to result in reduced p53 response in cultured cells ${ }^{8,12}$ consistent with the observed increase in the number of senescent cells, immunoblots using liver tissue lysates indicated higher expression of p53

a

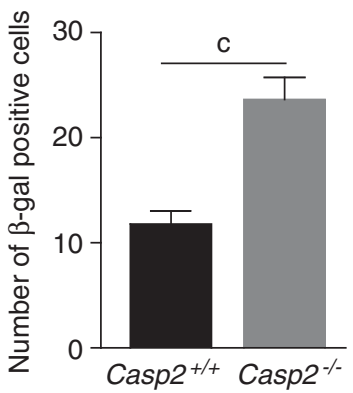

b
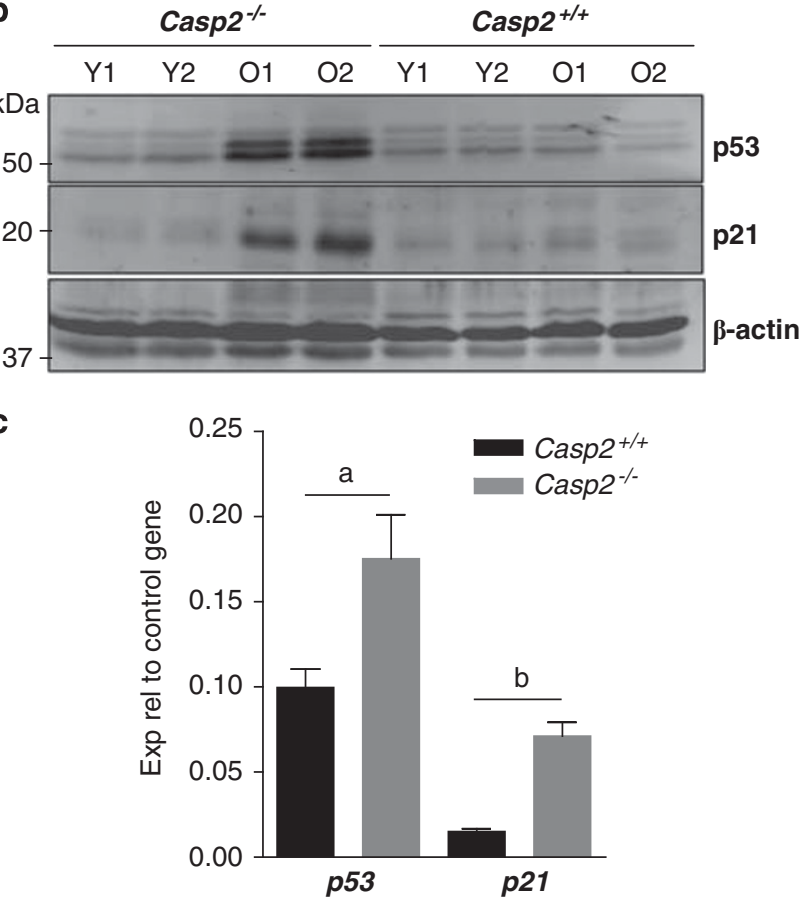

Figure 3 Old Casp2 $2^{-1-}$ mice show increased number of senescent cells and higher p53 and p21 expression. (a) $\beta$-Gal stained cells in liver sections from old Casp2 $^{+1+}$ and Casp2 $^{-1-}$ mice were enumerated by light microscopy. Values are average number of $\beta$-gal positive cells per field of view at $\times 40$ magnification. Results are mean \pm S.E.M. $(n=4),{ }^{c} P<0.001$. (b) Immunoblots showing higher expression of p53 and p21 in old Casp2 ${ }^{-1-}$ mice liver. $Y=$ young, $O=$ old. (c) Real-time PCR analysis showing increased transcript levels of p53 and p21 in old Casp $^{-1-}$ mice livers. Values represent mean \pm S.E.M. from three independent experiments each performed with three mice per group in triplicate. ${ }^{\mathrm{a}} P<0.05$ and ${ }^{\mathrm{b}} P<0.01$ represent comparison between the two groups and its key target p21 in old casp2 $2^{-1-}$ mice (Figure 3b). An increase in p53 protein was also noticed in kidney and spleen lysates from these animals (Supplementary Figure S4). In addition, increased p53 and p21 transcript levels were observed in the aged $\operatorname{casp}^{-1-}$ mice liver (Figure 3c). This suggests that the increased ROS observed in casp2 ${ }^{-1-}$ mice is associated with an accumulation of p21 and cellular senescence in older animals. Interestingly, we have observed that the levels of caspase-2 in wild-type mice are reduced in old animals (Supplementary Figure S4), further indicating that downregulation of caspase2 correlates with the ageing process.

Caspase-2 deficiency results in reduced FoxO expression and activity. We next assessed the levels of signalling proteins that are involved in the oxidative stress response pathway. One of the major family of transcription factors associated with ageing and stress resistance is FoxO. Examination of the expression of FoxO1 and FoxO3a genes in liver lysates indicated that both were significantly reduced in aged casp2 ${ }^{-1-}$ mice compared with the wild-type mice (Figure 4a). Total FoxO1 and $3 a$ protein levels were also reduced in older casp $2^{-1-}$ mice (Figure 4c). We next assessed the expression levels of FoxO target genes, MnSOD (SOD2), GSH-PX and catalase in liver and found that catalase and SOD2 were both downregulated in the old casp $^{-1-}$ mice, whereas GSH-PX expression was unchanged (Figure 4b).

To check if the loss of caspase-2 directly affects FoxO levels, we tested FoxO expression after caspase-2 knockdown in U2OS cells using siRNA. Although FoxO1 levels were not significantly reduced, we found reduced expression of FoxO3a and SOD2 (Figure 4d). This was further validated in knockdown experiments using IMR90 E1A-transformed cells (Supplementary Figure S5) and in primary MEFs isolated from wild-type and casp2 ${ }^{-1-}$ mice transfected either with GFP or caspase-2-GFP construct (Figures $4 e$ and $f$ ). Expression of caspase-2-GFP in these primary MEFs restored or enhanced the expression of both FoxO1 and FoxO3a expression at both transcript and protein levels (Figures $4 \mathrm{e}$ and $\mathrm{f}$ ). The expression of caspase-2-GFP did not alter SOD2 or catalase expression in wild-type cells but restored and further enhanced transcript levels in casp2 ${ }^{-1-}$ cells (Figure $4 \mathrm{~g}$ ). $G S H-P x$ expression also increased in both wild-type and casp2 $^{-1-}$ MEFs following caspase-2-GFP expression. These experiments indicate that caspase-2 differentially regulates the expression of two members of the FoxO family and its loss results in altered expression of some FoxO target genes.

Reduced expression of p53 target genes involved in stress resistance. FoxO and $\mathrm{p} 53$ are both $\mathrm{ROS}$-activated transcription factors that control mammalian sestrins (Sesns) gene transcription. ${ }^{13,14}$ Sesns are highly conserved proteins that accumulate in cells exposed to stress. In vitro, they exhibit oxidoreductase activity and function as antioxidants. In particular, they are involved in the regeneration of peroxiredoxins (Prdxs). Independently of their redox activity, Sesns lead to AMPK-dependent inhibition of TOR signalling and link genotoxic stress to TOR regulation. ${ }^{13}$ As both p53 and FoxO expression levels are aberrant in 


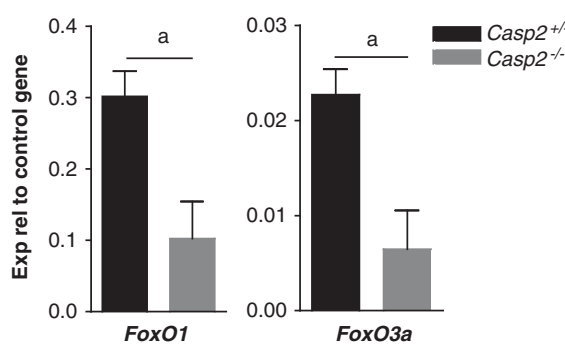

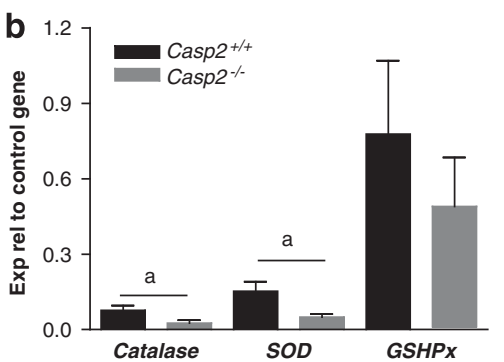

C
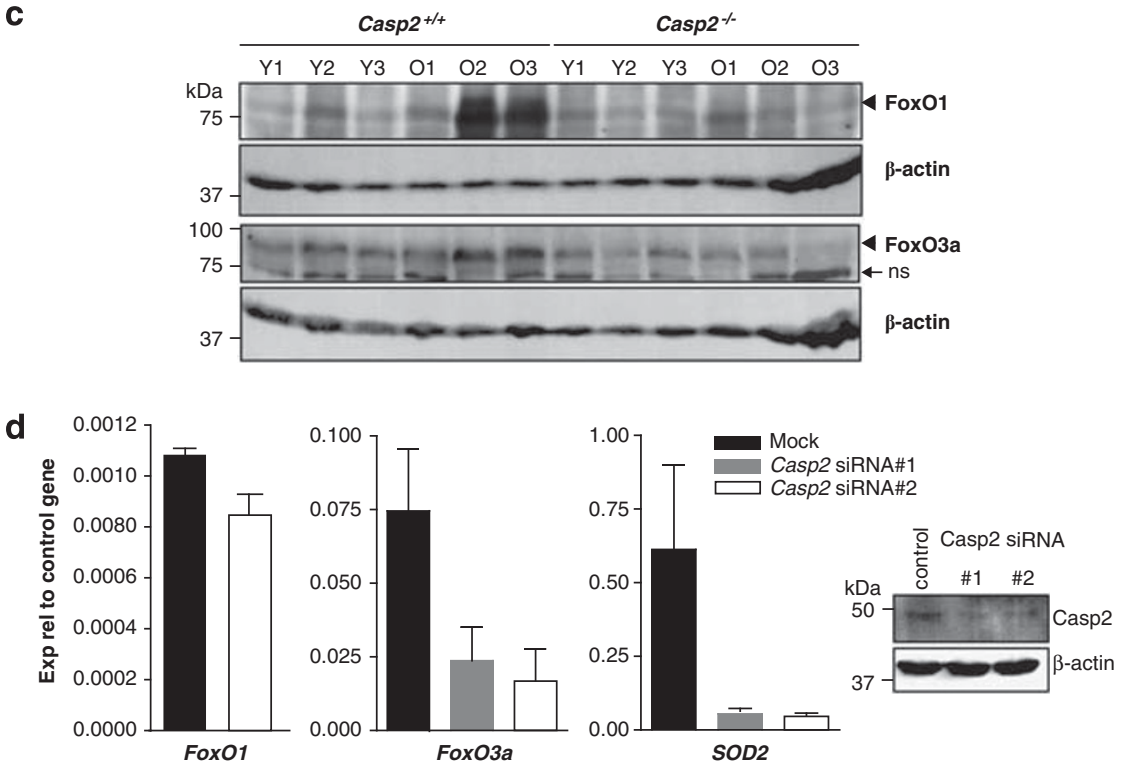

e
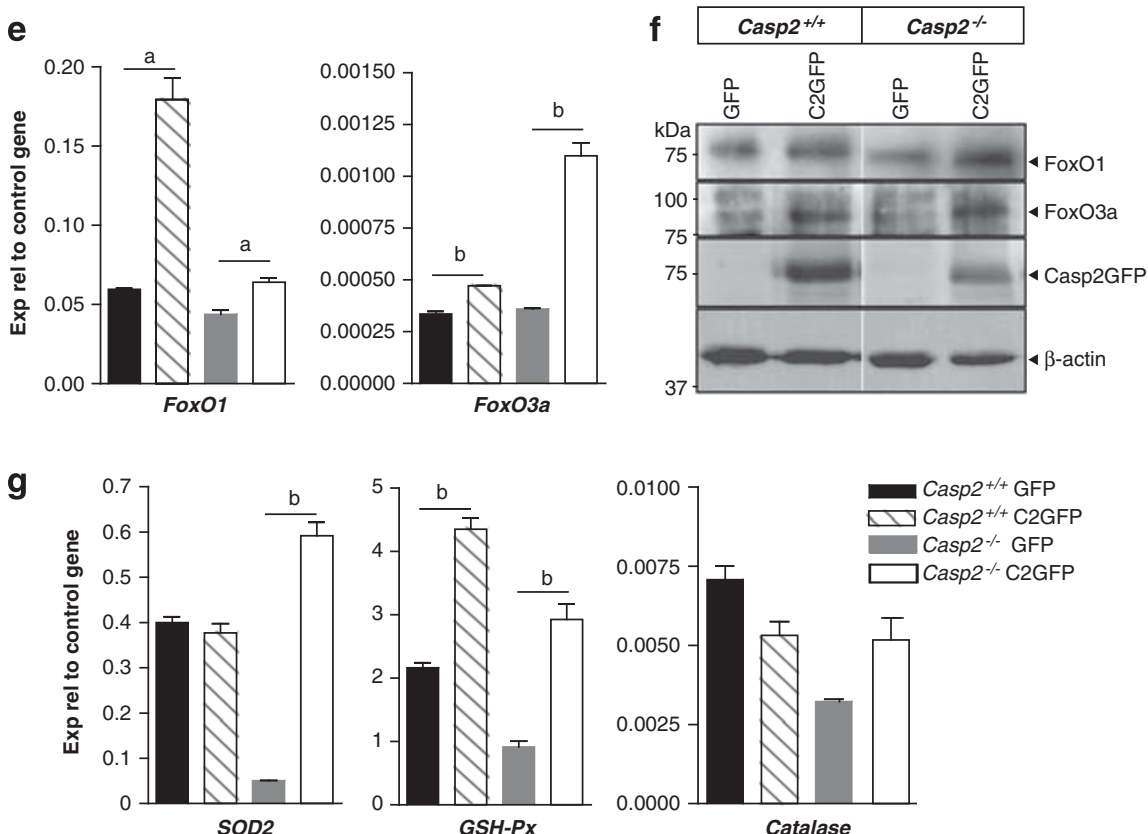

Figure 4 Old Casp2 ${ }^{-1-}$ mice have reduced FoxO expression. (a) Real-time PCR analysis showing decreased expression of FoxO1 and FoxO3a in old Casp2 ${ }^{-1-}$ mice liver. Values are mean \pm S.E.M. from three mice of each genotype, ${ }^{a} P<0.05$. (b) Expression of FoxO target genes, catalase and SOD2 was reduced, while no significant change was observed in GSH-Px levels. (c) Immunoblots showing lower expression of FoxO1 and FoxO3a in old Casp2 ${ }^{-1-}$ mice liver than in old Casp2 ${ }^{+/+}$liver. (d) Caspase-2 siRNA knockdown in U2OS cells leads to reduced expression of FoxO3a and its target gene (SOD2). (e and f) Re-expression of caspase-2-GFP in Casp2 ${ }^{+/+}$ and Casp2 ${ }^{-1-}$ MEFs increases expression of FoxO1 and FoxO3a transcripts and protein compared with the GFP vector control. (g) Increase in SOD2 and GSH-Px expression and restoration of catalase expression in Casp2 ${ }^{-1-}$ MEFs following caspase-2-GFP expression. Values are mean \pm S.E.M. from three independent experiments. ${ }^{\mathrm{a}} \mathrm{P}<0.05$ and ${ }^{\mathrm{b}} \mathrm{P}<0.01$ represents comparison between the groups 

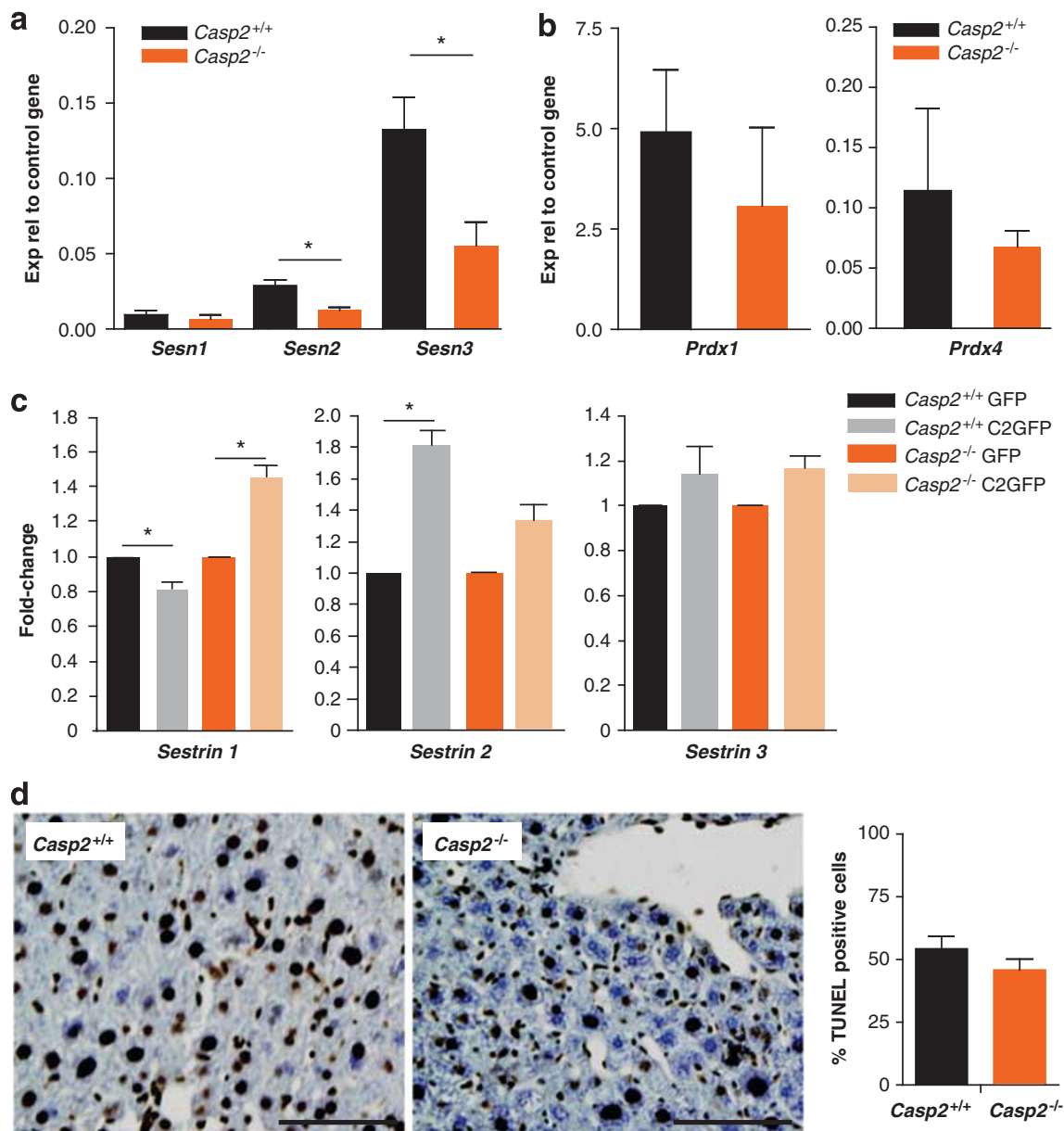

Figure 5 Old Casp2 ${ }^{-1-}$ mice show reduced expression of Sestrin antioxidant enzymes in liver. (a) Sestrin 2 and 3 expression decreases in aged Casp2 ${ }^{-1}$ mice. (b) No change in peroxiredoxin 1 and 4 expression was observed. (c) Re-expression of caspase-2-GFP in MEFs restores sestrin transcript levels. Values represent mean \pm S.E.M. from two independent experiments each performed with three mice per group in triplicate. ${ }^{*} P<0.01$ represents comparison between the groups. (d) Representative images of TUNEL on liver sections of old mice $(\times 20)$ and quantitation of frequency of TUNEL positive cells. Scale bar $=100 \mu \mathrm{m}$

caspase-2-deficient cells, we next assessed their transcriptional targets, Sesn1-3. Although Sesn1 expression remained unchanged, both Sesn2 and 3 were significantly reduced in old casp2 $2^{-1-}$ mice liver lysates (Figure 5a). Furthermore, the expression of caspase-2-GFP in these primary MEFs restored or enhanced the expression of Sesn 1-3 (Figure 5c). In contrast, Prdx 1 and 4 expression was unaffected (Figure $5 \mathrm{~b}$ ). The reduced expression of antioxidant genes likely explains the loss of enzyme activity seen previously and the observed increased in ROS levels following loss of caspase-2.

Given that loss of caspase-2 leads to increased ROS and differential regulation of antioxidant enzyme levels, we next assessed whether the increased ROS was associated with mitochondrial dysfunction. However, there was no apparent differences in mitochondrial membrane potential assessed by JC1 staining or in ATP levels in casp2 $2^{+/+}$and casp2 ${ }^{-1-}$ MEFs following menadione treatment (Supplementary Figure S6), indicating a possible role for caspase-2 downstream of mitochondria in the oxidative stress response.
Casp2 $^{-1-}$ animals show reduced ability to tolerate stress. As increased ROS can induce apoptosis, we carried out TUNEL staining to ascertain the extent of cell death in livers of aged wild-type and $\operatorname{casp}^{-1-}$ animals. The percentage of TUNEL positive cells in the old casp2 ${ }^{-1-}$ animals was not significantly different to that of wild-type mice (Figure 5d).

To assess the susceptibility of young mice to oxidative stress in vivo, we challenged mice by feeding them a diet containing $5 \%$ ethanol for 5 weeks. Both wild-type and casp $^{-1-}$ ethanol diet fed mice showed increased fat deposition (steatosis) in the liver accompanied by increased alanine transaminase (ALT) activity, a marker of liver damage (Figures $6 \mathrm{a}-\mathrm{c}$ ). However, there was no difference between the wild-type and casp2 $2^{-1-}$ mice. Remarkably, during the short 5-week feeding schedule the wild-type animals on ethanol diet showed upregulation in GSH-Px and SOD activity (Figures $6 \mathrm{~d}$ and e). In contrast, casp2 ${ }^{-1-}$ animals failed to respond to the oxidative stress, as GSH-Px, SOD activities were comparable to control mice (Figures $6 d$ and e). These 
a
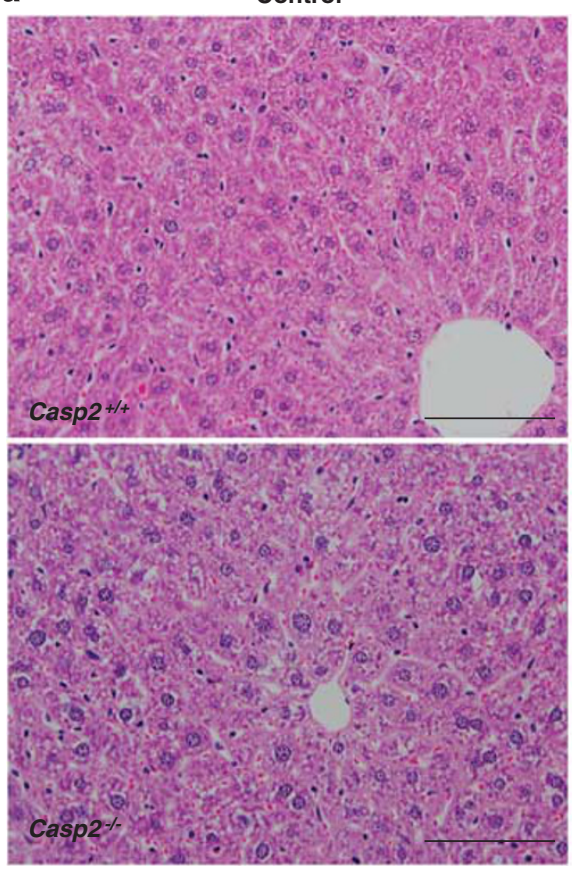

b
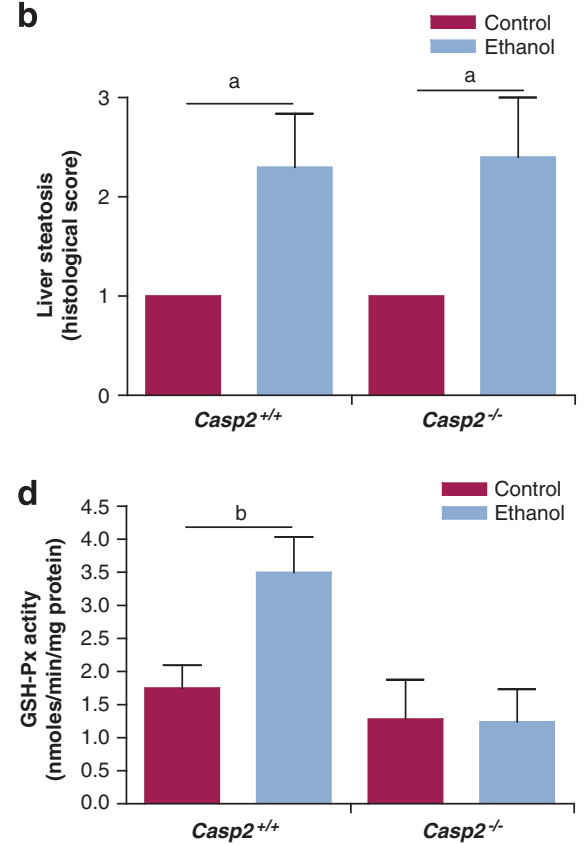
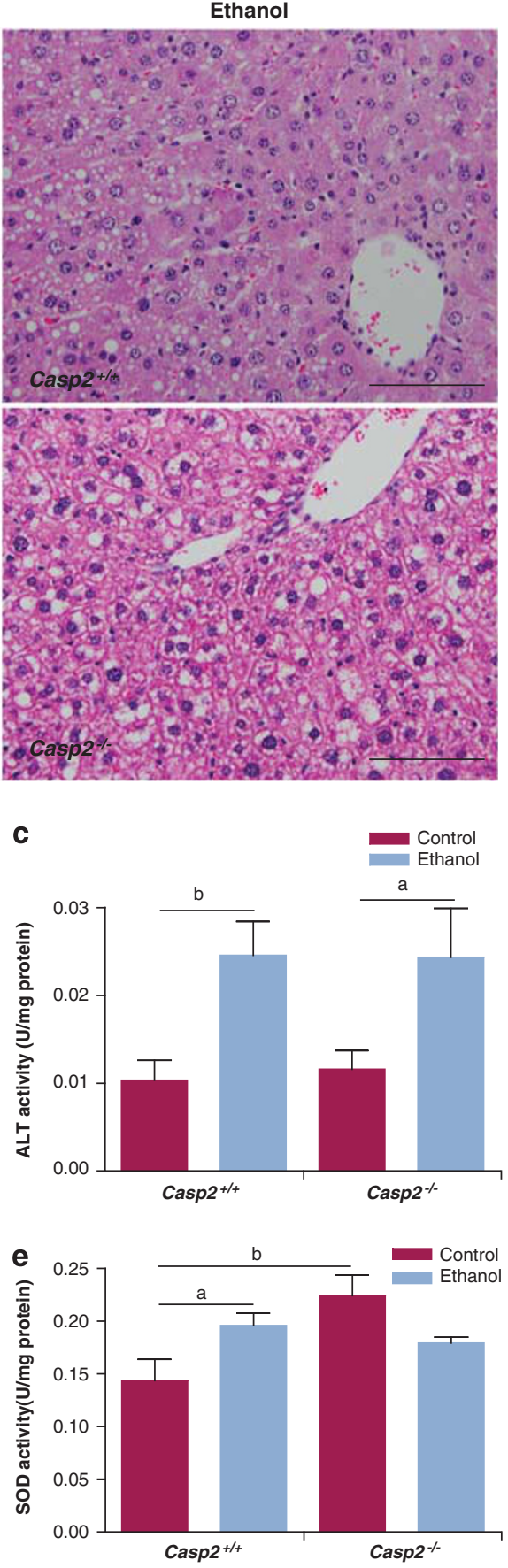

Figure 6 Oxidative stress and ROS in ethanol diet fed mice. (a) Representative $\mathrm{H}$ and $\mathrm{E}$ stained liver sections from control or ethanol diet fed mice $(\times 20)$, scale bar $=100 \mu \mathrm{m}$. (b) Scoring liver steatosis in the different treatment groups $(n=5)$. (c) Serum ALT levels in Casp2 ${ }^{+/+}$and Casp2 ${ }^{-1-}$ mice fed either control diet or diet containing $5 \%$ ethanol. (d) GSH-Px activity is unchanged in the ethanol diet fed Casp2 ${ }^{-1-}$ mice. (e) SOD activity is unchanged in the ethanol diet fed Casp2 ${ }^{-1-}$ mice. Values are mean \pm S.E.M. $(n=5) .{ }^{\mathrm{a}} P<0.05$ and ${ }^{\mathrm{b}} P<0.01$ represents comparison between the groups

data indicate that casp2 $2^{-1-}$ mice are unable to respond to ethanol toxicity-induced oxidative damage and may therefore be more susceptible to succumbing to oxidative stress over time.

Interestingly, a further increase in the number of TUNEL positive cells in liver was observed in casp $^{-1-}$ animals compared with the wild-type liver after ethanol intoxication (Figures $7 \mathrm{a}$ and b). These experiments suggest that caspase-2 itself is not required for apoptosis induced by increased ROS burden and oxidative damage in vivo.

\section{Discussion}

The present study indicates that caspase-2 is crucial for cellular stress signalling by regulating ROS levels. In the absence of caspase-2, mice display a weakened response to 
a
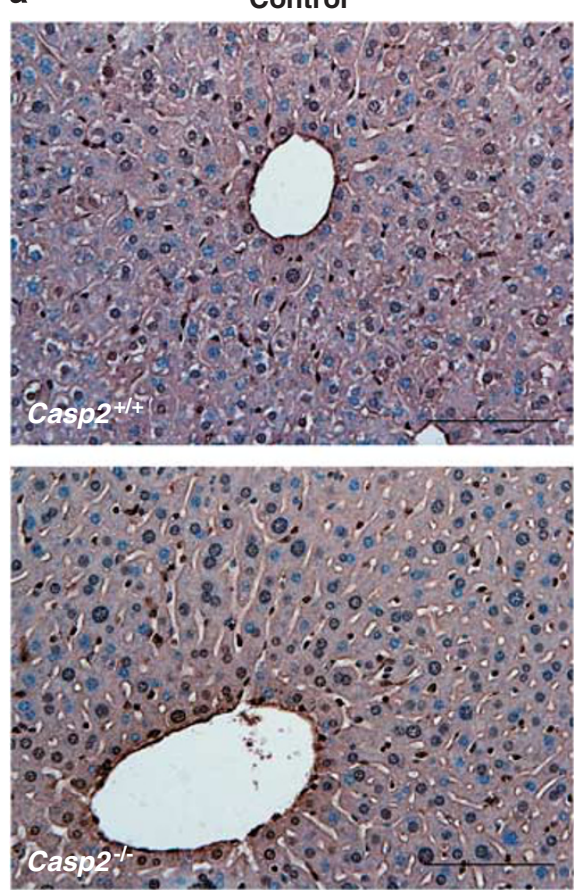

Ethanol
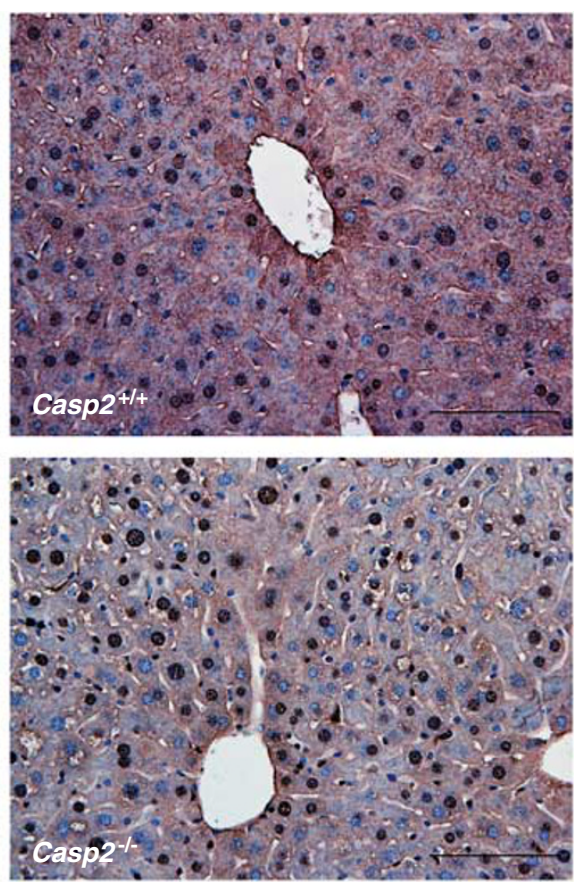

b

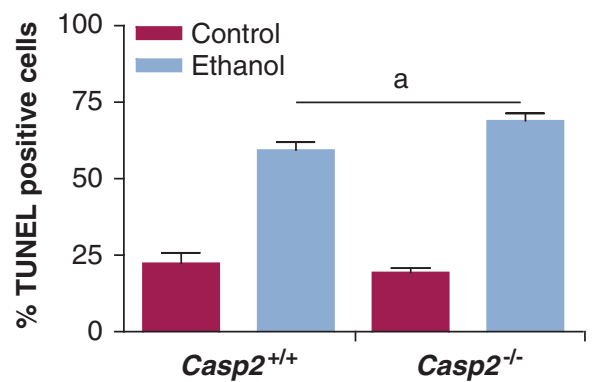

Figure 7 TUNEL in ethanol diet fed mice liver. (a) Representative images of TUNEL stained liver sections from each group $(\times 20)$, scale bar $=100 \mu \mathrm{m}$ and (b) quantitation of frequency of TUNEL positive cells, are shown. Values are mean \pm S.E.M. from three animals. ${ }^{\mathrm{a}} P<0.05$

tolerate cellular stress and increased oxidative damage. We show that increased ROS and oxidative damage in the absence of caspase- 2 contribute to a premature ageing phenotype in these mice. ${ }^{7}$ Importantly, our data suggest that this increased oxidative stress is partly because of the reduced activity of antioxidant enzymes SOD, GSH-Px, and antioxidants such as sestrins, which is likely caused by reduced FoxO expression and an increased p53 response in aged mice.

We found that the maximum lifespan of our casp2 ${ }^{-1-}$ animals was significantly reduced consistent with previous data with a different caspase-2 knockout line. ${ }^{7}$ These mice also displayed several early ageing-related phenotypes. However, it is important to note that while caspase-2 deficiency leads to signs of early ageing, the median lifespan of these animals is not significantly different from their wildtype littermates.

As caspase-2 is linked to stress-associated apoptosis, and increased oxidative stress is implicated in ageing, we measured several markers of oxidative damage in caspase2-deficient animals. The old animals showed higher lipid peroxidation compared with the young mice and this was more pronounced in the old casp2 $2^{-1-}$ animals compared with their wild-type counterparts. Protein oxidation was also markedly increased in the old casp2 $2^{-/}$animals. On the other hand, the activities of GSH-Px and SOD, the key antioxidant enzymes involved in ROS removal were diminished in these animals. This reduced activity explains the increased ROS and increased oxidative damage observed in the old casp2 $2^{-1-}$ animals. Interestingly, while the young mice did not display significantly altered enzyme activity, protein oxidation was significantly higher in the young mice (Supplementary Figure S3b). This may be due to the limited sensitivity of some of the assays used (enzyme activity in young mice was very low). The increased protein carbonyls in the age-matched young casp2 $2^{-1-}$ animals indicates that these mice experience increased oxidative stress and highlights a role of caspase-2 in this process.

Increased DNA damage is frequently associated with ageing $^{21}$ and DNA is most vulnerable to attack by free radicals. Oxidative damage to DNA showed an age-dependent increase in both wild-type and $\operatorname{casp}^{-1-}$ mice. Again, old 
casp $2^{-1-}$ animals accumulated significantly more oxidative damage compared with the old wild-type animals. Caspase-2 has been implicated in cell cycle checkpoint regulation, as casp2 $^{-1}$ MEFs fail to fully arrest in response to $\gamma$-irradiation. ${ }^{8}$ This would lead to inefficient repair of DNA and accumulation of damaged DNA as cells continue to cycle. Inability to efficiently repair DNA damage and increased frequency of DNA breaks are commonly seen in several premature ageing mouse models. ${ }^{22,23}$ We noticed increased numbers of $\gamma-\mathrm{H} 2 \mathrm{AX}$ positive hepatocytes in the old casp2 $2^{-1-}$ animals. In addition casp2 $2^{-1-}$ MEFs challenged with menadione displayed a higher proportion of $\gamma$-H2AX positive nuclei. These experiments suggest that the absence of caspase-2 disrupts the normal antioxidant defence thereby increasing ROS-induced DNA damage.

To identify the mechanism behind the altered enzyme activity and increased oxidative stress in casp2 $2^{-1-}$ mice, we assessed the transcriptional levels and activity of the stress responsive transcription factor, p53 that has been associated with caspase-2 in several studies. ${ }^{2}$ We observed increased p53 and p21 expression in old casp2 ${ }^{-1-}$ mice livers. These results seem somewhat contrary to in vitro data. ${ }^{8,12}$ However, it must be noted that the activation of p53 response was observed only in the old casp2 $2^{-1}$ mice and not in young animals. This may be an adaptive response to limit damage and suggests involvement of other mechanisms such as the altered redox status of cells/tissues and is perhaps not a direct consequence of the loss of caspase-2. It was also observed that while p53 levels increased, its target p21 showed a tissuespecific response, while some others such as sestrins and other antioxidant genes were downregulated. Further studies are required to address the role of caspase- 2 in p53 regulation.

Increased p53 levels have been linked to cellular stress responses in ageing mice..$^{24,25} \mathrm{p} 53$ activates several genes to overcome the consequences of stress by regulating cell viability, growth, proliferation, repair and metabolism. Among the many antioxidant genes activated by p53, sestrins are critical for suppression of ROS and protection from oxidative stress, transformation and genomic instability. ${ }^{13}$ Both Sesn 1 and Sesn2 are stress responsive genes that confer resistance to oxidative stress both in vivo and in vitro. ${ }^{13,26}$ We observed reduced Sesn2 and Sesn3 expression in the old casp2 ${ }^{-1}$ mice, but Sesn 1 levels were unchanged. Sestrins can regulate ROS through their direct effect on regenerating Prdx proteins that reduce ROS. ${ }^{13}$ Our data indicate that regulation of Prdx gene expression, which increases following oxidative stress, is not affected in casp2 $2^{-1-}$ mice. On the other hand all three Sesn family members are also activated by FoxO proteins.

We found that FoxO1 and 3 a expression was also reduced in the old casp2 $2^{-1-}$ animals. Similar to $\mathrm{p} 53$, FoxO proteins are activated by cellular stress and in turn regulate genes involved in cell cycle, metabolism, antioxidant defence and DNA repair. ${ }^{27}$ Previous studies indicate that they have a critical role in regulating longevity. ${ }^{14}$ Among all sestrin proteins, Sesn3 is reported to be the most affected by FoxO and the ability of FoxO to elevate Sesn3 RNA and protein is conserved in rodent and human cells. ${ }^{17}$ Sesn3 overexpression was shown to reduce ROS induced by activated Ras. ${ }^{28}$ Furthermore, the inactivation of Sestrin in flies causes accumulation of age-associated damage. ${ }^{29}$ Among other targets of FoxO are the antioxidant enzymes SOD2 and catalase. The expression and activity of both these enzymes was reduced in the old casp2 ${ }^{-1-}$ mice. The absence of FoxOinduced antioxidant enzymes and lower Sesn3 expression in the aged casp $2^{-1-}$ mice therefore likely contributes to the observed increased intracellular ROS and oxidative stress. Furthermore, our studies with caspase-2 re-expression in casp2 $^{-1-}$ MEFs as well as siRNA-mediated ablation, indicate that caspase-2 has a critical role in regulating the levels of FoxO and its target genes. These findings suggest that caspase-2 functions to modulate ROS levels through the regulation of FoxO expression.

Our ethanol feeding experiments show that casp2 ${ }^{-1-}$ mice do not upregulate SOD and GSH-Px activities normally seen in the wild-type animals. This indicates that even during the short feeding schedule, casp2 $2^{-1}$ mice have decreased sensitivity to ethanol-induced ROS and toxicity as they fail to upregulate their antioxidant defence in response to excess free radicals. This presumably results in enhanced cellular damage, which was evident from an increase in the number of TUNEL positive cells in casp2 $2^{-1-}$ livers.

Metabolic and mitochondrial dysfunction are associated with increased oxidative stress, and a function of caspase- 2 in metabolic regulation and cell death in Xenopus oocytes has been demonstrated. ${ }^{30}$ In particular, caspase- 2 is required for the regulation of the pentose phosphate pathway as an important antioxidant defence mechanism. ${ }^{30,31}$ However, it remains unclear whether this role would explain our observations in casp2 $2^{-1-}$ animals. Analysis of mitochondrial dysfunction in MEFs indicates that loss of caspase-2 does not affect mitochondrial membrane potential or respiration during the oxidative stress response. It would, however, be important to assess the role of mitochondria in aged casp2 ${ }^{-1-}$ animals to determine any possible association between caspase-2, ROS and mitochondria.

In summary, our study demonstrates the importance of caspase-2 in sensing changes in cells' redox environment and initiating suitable adaptive responses to regulate ROSmediated oxidative stress. Although the exact mechanism of caspase-2-mediated ROS regulation requires further studies, the work reported here provides a novel link between caspase-2 and oxidative stress regulation, mediated at least in part via FoxO transcription factors. Continued exposure to stress, for example, over the animal's lifetime, would result in the accumulation of damaged cells that can potentially lead to a shorter lifespan. Discovery of specific caspase-2 targets, which has been challenging so far due to low substrate specificity between caspases, will be necessary to demonstrate the precise mechanism by which caspase- 2 regulates the oxidative stress response.

\footnotetext{
Materials and Methods

Mice and MEFs. Casp2 ${ }^{-1-}$ mice ${ }^{6,32}$ were maintained in a pathogen-free barrier facility. These mice have been backcrossed onto C57BL/6J mice for at least 12 generations. All animals were housed at the SA Pathology Animal Facility and treated in accordance with the protocols approved by the Animal Ethics Committee. For ageing studies, the animals were monitored for 24-26 months (old mice), while mature animals at 2-3 months were used as young mice. Survival curves were generated by the Kaplan-Meier method using Graphpad Prism software (GraphPad Inc., La Jolla, CA, USA), and Fishers Exact test was used to analyze survival data. Primary MEFs were derived embryos at day 13.5 as described earlier ${ }^{32,33}$ and
} 
grown at $37^{\circ} \mathrm{C}$ with $10 \% \mathrm{CO}_{2}$ in high glucose DMEM, supplemented with $0.2 \mathrm{mM}$ L-glutamine, $10 \mathrm{mM}$ HEPES, 10\% fetal bovine serum (JRH Biosciences, Brooklyn Victoria, Australia), 0.5\% $\beta$-mercaptoethanol, non-essential amino acid mix (SigmaAldrich, St. Louis, MO, USA) and $100 \mu \mathrm{M}$ penicillin/Streptomycin mix (CSL Biosciences, Parkville, Victoria , Australia).

Histology. Immediately after euthanizing animals, organs were removed and either snap frozen in liquid nitrogen and stored at $-70^{\circ} \mathrm{C}$ or fixed in formalin. The fixed specimens were embedded in paraffin, sectioned $(7 \mu \mathrm{m})$ and stained with haematoxylin and eosin. For bone volume measurements, femurs from old wild-type and casp2 ${ }^{-1-}$ mice were scanned by microCT (Adelaide Microscopy, University of Adelaide, Adelaide, South Australia, Australia).

Skin thickness analysis. Skin sections stained with haematoxylin and eosin, were visualized under the light microscope. The epidermal thickness was measured from stratum basale to stratum granulosum (excluding stratum corneum), derma thickness was the distance between the epidermis and the hypodermis (SAT), SAT thickness was measured as the distance between the dermis and the muscle layer (panniculus carnosus). In every section, five measurements of the distances between the indicated lines were measured at different randomly selected positions and the average thickness of $n=4-5$ sections for each genotype were calculated.

Antioxidant enzyme activity. Activity of antioxidant enzymes such as SOD and GSH-Px was determined by using commercially available kits according to manufacturer's instructions (Cayman Chemicals, Ann Arbor, MI, USA). Lipid peroxidation was measured using the TBARS assay kit and protein oxidation was measured using protein carbonyl kit (Cayman Chemicals). Protein concentration was determined using the Biorad Protein Assay reagent (Bio-Rad, Gladesville, New South Wales, Australia).

Detection of intracellular ROS. The intracellular ROS levels were monitored by flow cytometry using $\mathrm{CM}-\mathrm{H}_{2} \mathrm{DCFDA}$ (Invitrogen, Carlsbad, CA USA). Immortalized MEFs derived from the wild-type and caspase-2 ${ }^{-1-}$ animals were either untreated or treated with a superoxide generator menadione $(10 \mu \mathrm{M})$ for $6 \mathrm{~h}$. The cells were washed and again incubated with $\mathrm{CM}-\mathrm{H}_{2}$ DCFDA for $30 \mathrm{~min}$ after which the cells were trypsinized and analyzed by flow cytometry.

Immunoblotting. Liver homogenates were obtained by mincing liver pieces in RIPA buffer containing protease inhibitors (Roche, Indianapolis, IN, USA). Homogenates were clarified by centrifugation at $13.2 \mathrm{k}$ r.p.m. and protein concentration determined by the Bradford method. For immunoblot analysis, $50 \mu \mathrm{g}$ of lysates were resolved on SDS-PAGE and transferred onto PVDF membrane and probed with the antibody of interest. Proteins were visualized using ECF (Amersham, Sunnyvale, CA, USA). The following antibodies were used: p53, Fox01, FoxO 3a (Cell Signaling, Danvers, MA, USA), p21 (Santa Cruz Biotechnology, Inc., Santa Cruz, CA, USA) caspase-2 (clone 11B4) and $\gamma$-H2AX (Millipore, Billerica, MA, USA), $\beta$-actin (Sigma-Aldrich).

qPCR analysis. Total RNA isolated by Trizol reagent (Invitrogen) was used for cDNA using high capacity cDNA transcription kit (Applied Biosciences, Foster City, CA, USA) and random primers. Real-time PCR was performed on a Rotor-Gene 3000 (Corbett Research, Mortlake, New South Wales, Australia) using $\mathrm{RT}^{2}$ SYBR Green/Rox PCR Master Mix (SA BioSciences, Frederick, MD, USA) using primers pairs listed in Supplementary Table S1. Reactions were performed in triplicates and the mRNA expression was normalized against $\beta$-actin as an internal control using the $2^{-\Delta \mathrm{CT}}$ method and $2^{-\Delta \Delta \mathrm{CT}}$ for determining fold-change.

Detection of $\gamma-\mathrm{H} 2 \mathrm{AX}$ foci. Paraffin-embedded liver sections from old wildtype and caspase- $2^{-1-}$ mice were deparaffinized in xylene and rehydrated in descending grades of alcohol. Endogenous peroxidase activity was blocked with $0.3 \% \mathrm{H}_{2} \mathrm{O}_{2}$. Sections were blocked with $3 \% \mathrm{FCS}$ in PBS. Slides were incubated with antibody to $\gamma-\operatorname{H} 2 A X\left(1: 50\right.$; Millipore) overnight at $4{ }^{\circ} \mathrm{C}$. This was followed by incubation with anti-rabbit biotin $(1: 100 ; 1 \mathrm{~h})$ and subsequent incubation with $A B C$ reagent (Vectastain, Vector Laboratories, Burlingame, CA, USA). DAB was used as substrate. The sections were then counter stained in haematoxylin and mounted using DPX. For immunostaining MEFs, cells were fixed using ice-cold methanol. The DSB foci were detected by immunostaining with a monoclonal antibody to $\gamma$ $\operatorname{H2AX}$ (1:100; Millipore) followed by incubation with anti rabbit-Alexa fluor-532 $(1: 400)$ as secondary antibody.
Caspase-2 knockdown and knockin. Casp2-GFP construct and a vector control (pEGFP-N1) (2 $\mu \mathrm{g}$ each) were transfected into Casp2 $2^{+/+}$or Casp2 $^{-1-}$ MEFs using lipofectamine LTX reagent (Invitrogen). To minimize cell death $4 \mathrm{~h}$ before transfection $50 \mu \mathrm{M} z-V A D$ was added. Cells were incubated with the transfection complexes for 16-18 $\mathrm{h}$ and then harvested for subsequent analysis. Caspase-2 was transiently knocked down in IMR90-E1A and U2OS cells using two different sets of siRNA in order to minimize any potential off-target effects. Sequences of the siRNA used: siRNA no. $15^{\prime}$-ACAGCUGUUGUUGAGCGAAd TdT-3'; siRNA no. 2 5'-UUGUUGAGCGAAUUGUUATT-3'. The transfections were carried out using Trans IT-TKO transfection reagent (Mirus Bio LLC, Madison, WI, USA) for $48 \mathrm{~h}$ according to manufacturer's instructions.

Senescence-associated $\boldsymbol{\beta}$-gal staining. Senescence $\beta$-galactosidase staining kit (Cell Signalling) was used to identify senescent cells. Briefly, $7 \mu \mathrm{m}$ thick liver cryosections were fixed using the fixative provided in the kit for $10 \mathrm{~min}$ and then rinsed thoroughly in PBS. The slides were incubated with the staining solution for $18-20 \mathrm{~h}$ at $37^{\circ} \mathrm{C}$. The stained sections were washed with PBS, counterstained with haematoxylin and mounted in glycerol.

Measurement of ATP. Cells $\left(2 \times 10^{5}\right)$ were seeded onto $60 \mathrm{~mm}$ tissue culture dishes and cultured overnight $(16 \mathrm{~h})$. Media was changed and cells treated with or without menadione $(10 \mu \mathrm{M})$ for $6 \mathrm{~h}$. Intracellular ATP levels were determined in trypsinized cells using the ATP Bioluminescent Somatic Cell Assay Kit (Sigma-Aldrich).

Mitochondrial membrane potential $\left(\psi_{\mathrm{m}}\right)$. Changes in mitochondrial membrane potential $\left(\psi_{\mathrm{m}}\right)$ were assessed by cell staining with JC-1 dye (Cayman Chemicals). Briefly, cells $\left(2 \times 10^{5}\right)$ were seeded onto glass coverslips in a six-well dish and cultured overnight. Media was changed and cells treated with or without menadione $(10 \mu \mathrm{M})$ for $6 \mathrm{~h}$. Live cells were stained with $\mathrm{JC}-1(50 \mu \mathrm{g} / \mathrm{ml})$ for $30 \mathrm{~min}$ and imaged by fluorescence microscopy.

Ethanol diet studies. Eight- to twelve-week-old ( $25 \mathrm{~g}$ initial body weight) matched wild-type and casp2 ${ }^{-1-}$ mice were either fed Lieber and Decarli diet (Able Scientific, Canning Vale, WA, USA) containing 5\% ethanol or a diet balanced iso-calorically with maltose for a period of 5 weeks. Mice were monitored daily and at the end of the diet-feeding schedule mice were euthanized by $\mathrm{CO}_{2}$ asphyxiation, and blood and tissues collected for further analysis. Serum ALT levels were measured using ALT kit (Cayman Chemicals).

TUNEL. Cells with DNA breaks were measured using TUNEL (POD) kit as per the manufactures instructions (Roche)

Statistical analysis of data. Graphpad Prism software (GraphPad Inc.) was used for statistical analysis. Fisher's Exact test and the Boschloo's test were used to analyze the survival data, and student's $t$-test was used for all other analysis unless otherwise stated. Data are expressed as mean \pm S.D. for all animal analysis and mean \pm S.E.M. $P<0.05$ was considered significant.

\section{Conflict of Interest}

The authors declare no conflict of interest.

Acknowledgements. We thank David Vaux for caspase-2 knockout mice, Andreas Villunger for SV40 immortalized MEFs, David Huang for advice, Nancy Briggs (University of Adelaide) for statistical advice and staff at the SA Pathology animal facility for help in maintaining the mouse strains. This work was supported by the National Health and Medical Research Council of Australia project Grants (626905 and APP1021456) and a Senior Principal Research Fellowship (1002863) to SK

1. Kumar S. Caspase function in programmed cell death. Cell Death Differ 2007; 14: 32-43.

2. Kumar S. Caspase 2 in apoptosis, the DNA damage response and tumour suppression: enigma no more? Nat Rev Cancer 2009; 9: 897-903

3. Kumar S, Kinoshita M, Noda M, Copeland NG, Jenkins NA. Induction of apoptosis by the mouse Nedd2 gene, which encodes a protein similar to the product of the Caenorhabditis elegans cell death gene ced-3 and the mammalian IL-1 beta-converting enzyme. Genes Dev 1994; 8: 1613-1626. 
4. Kumar S, Tomooka Y, Noda M. Identification of a set of genes with developmentally down-regulated expression in the mouse brain. Biochem Biophys Res Commun 1992; 185: 1155-1161.

5. Bergeron L, Perez Gl, Macdonald G, Shi L, Sun Y, Jurisicova A et al. Defects in regulation of apoptosis in caspase-2-deficient mice. Genes Dev 1998; 12: 1304-1314.

6. O'Reilly LA, Ekert P, Harvey N, Marsden V, Cullen L, Vaux DL et al. Caspase-2 is not required for thymocyte or neuronal apoptosis even though cleavage of caspase-2 is dependent on both Apaf-1 and caspase-9. Cell Death Differ 2002; 9: 832-841.

7. Zhang Y, Padalecki SS, Chaudhuri AR, De Waal E, Goins BA, Grubbs B et al. Caspase-2 deficiency enhances aging-related traits in mice. Mech Ageing Dev 2007; 128: 213-221.

8. Ho LH, Taylor R, Dorstyn L, Cakouros D, Bouillet P, Kumar S. A tumor suppressor function for caspase-2. Proc Natl Acad Sci USA 2009; 106: 5336-5341.

9. Hoeijmakers JH. DNA damage, aging, and cancer. N Engl J Med 2009; 361: 1475-1485.

10. Kohen R, Nyska A. Oxidation of biological systems: oxidative stress phenomena, antioxidants, redox reactions, and methods for their quantification. Toxicol Pathol 2002; 30 : 620-650.

11. Kenyon CJ. The genetics of ageing. Nature 2010; 464: 504-512.

12. Oliver TG, Meylan E, Chang GP, Xue W, Burke JR, Humpton TJ et al. Caspase-2-mediated cleavage of Mdm2 creates a p53-induced positive feedback loop. Mol Cell 2011; 43: 57-71.

13. Budanov AV. Stress-responsive sestrins link p53 with redox regulation and mammalian target of rapamycin signaling. Antioxid Redox Signal 2011; 15: 1679-1690.

14. Greer EL, Brunet A. FOXO transcription factors at the interface between longevity and tumor suppression. Oncogene 2005; 24: 7410-7425.

15. Zhao J, Brault JJ, Schild A, Cao P, Sandri M, Schiaffino S et al. FoxO3 coordinately activates protein degradation by the autophagic/lysosomal and proteasomal pathways in atrophying muscle cells. Cell Metab 2007; 6: 472-483.

16. Bouchard C, Lee S, Paulus-Hock V, Loddenkemper C, Eilers M, Schmitt CA. FoxO transcription factors suppress Myc-driven lymphomagenesis via direct activation of Arf. Genes Dev 2007; 21: 2775-2787.

17. Nogueira V, Park Y, Chen CC, Xu PZ, Chen ML, Tonic I et al. Akt determines replicative senescence and oxidative or oncogenic premature senescence and sensitizes cells to oxidative apoptosis. Cancer Cell 2008; 14: 458-470.

18. Tothova Z, Kollipara R, Huntly BJ, Lee BH, Castrillon DH, Cullen DE et al. FoxOs are critica mediators of hematopoietic stem cell resistance to physiologic oxidative stress. Cell 2007; 128: $325-339$
19. Burma S, Chen BP, Murphy M, Kurimasa A, Chen DJ. ATM phosphorylates histone H2AX in response to DNA double-strand breaks. J Biol Chem 2001; 276 $42462-42467$.

20. Wang C, Jurk D, Maddick M, Nelson G, Martin-Ruiz C, von Zglinicki T. DNA damage response and cellular senescence in tissues of aging mice. Aging Cell 2009; 8: 311-323.

21. Kenyon J, Gerson SL. The role of DNA damage repair in aging of adult stem cells. Nucleic Acids Res 2007; 35: 7557-7565.

22. de Boer J, Andressoo JO, de Wit J, Huijmans J, Beems RB, van Steeg $\mathrm{H}$ et al. Premature aging in mice deficient in DNA repair and transcription. Science 2002; 296: 1276-1279.

23. Burtner CR, Kennedy BK. Progeria syndromes and ageing: what is the connection? Nat Rev Mol Cell Biol 2010; 11: 567-578.

24. Hinkal GW, Gatza CE, Parikh N, Donehower LA. Altered senescence, apoptosis, and DNA damage response in a mutant p53 model of accelerated aging. Mech Ageing Dev 2009; 130: 262-271.

25. Rodier F, Campisi J, Bhaumik D. Two faces of p53: aging and tumor suppression. Nucleic Acids Res 2007; 35: 7475-7484.

26. Sablina AA, Budanov AV, llyinskaya GV, Agapova LS, Kravchenko JE, Chumakov PM. The antioxidant function of the p53 tumor suppressor. Nat Med 2005; 11: 1306-1313.

27. van der Horst A, Burgering BM. Stressing the role of FoxO proteins in lifespan and disease. Nat Rev Mol Cell Biol 2007; 8: 440-450.

28. Kopnin PB, Agapova LS, Kopnin BP, Chumakov PM. Repression of sestrin family genes contributes to oncogenic Ras-induced reactive oxygen species up-regulation and genetic instability. Cancer Res 2007; 67: 4671-4678.

29. Lee JH, Budanov AV, Park EJ, Birse R, Kim TE, Perkins GA et al. Sestrin as a feedback inhibitor of TOR that prevents age-related pathologies. Science 2010; 327: 1223-1228.

30. Nutt LK, Buchakjian MR, Gan E, Darbandi R, Yoon SY, Wu JQ et al. Metabolic control of oocyte apoptosis mediated by 14-3-3zeta-regulated dephosphorylation of caspase-2. Dev Cell 2009; 16: 856-866.

31. Andersen JL, Thompson JW, Lindblom KR, Johnson ES, Yang CS, Lilley LR et al. A biotin switch-based proteomics approach identifies 14-3-3zeta as a target of sirt1 in the metabolic regulation of caspase-2. Mol Cell 2011; 43: 834-842.

32. Ho LH, Read SH, Dorstyn L, Lambrusco L, Kumar S. Caspase-2 is required for cell death induced by cytoskeletal disruption. Oncogene 2008; 27: 3393-3404.

33. Lowe SW, Ruley HE, Jacks T, Housman DE. p53-dependent apoptosis modulates the cytotoxicity of anticancer agents. Cell 1993; 74: 957-967.

\section{Supplementary Information accompanies the paper on Cell Death and Differentiation website (http://www.nature.com/cdd)}

